$\mathbb{T}$ periodica polytechnica

\author{
Electrical Engineering \\ and Computer Science \\ $58 / 1(2014) 23+27$ \\ doi: 10.3311/PPee.6993
}

Creative Commons Attribution (1)

RESEARCH ARTICLE

\section{Derivation of Equations for Conductor and Sag Curves of an Overhead Line Based on a Given Catenary Constant}

\author{
Alen Hatibovic
}

Received 2013-08-01, revised 2013-09-15, accepted 2013-09-16

\begin{abstract}
When the spans of an overhead line are large (for instance over 400 metres) the conductor curve cannot be considered as a parabola, since in that case the difference in comparison to the catenary cannot be neglected. At such times the exact calculation has to be applied, i.e. the conductor curve has to be considered as a catenary (hyperbolic cosine). The catenary based calculation does not have limitations, it can be used for small and large spans as well, but in comparison to the parabola method it is significantly more complicated. This article shows the way of derivation of new equations for the conductor and sag curves based on a known catenary constant, which refers to the chosen conductor type, span length, tension and temperature of the overhead line. The shown formulas ensure exact computing of the conductor height and sag at any point of the span, avoiding errors generated by the approximation of the catenary by a parabola.
\end{abstract}

\section{Keywords}

overhead lines $\cdot$ catenary $\cdot$ sag $\cdot$ conductor curve $\cdot$ inclined span $\cdot$ levelled span

\section{Introduction}

The catenary based calculation is generally used for high voltage overhead line (OHL) design, but in case of a medium or low voltage network it is rarely done. A typical high voltage $\mathrm{OHL}$ is shown in Figure 1.

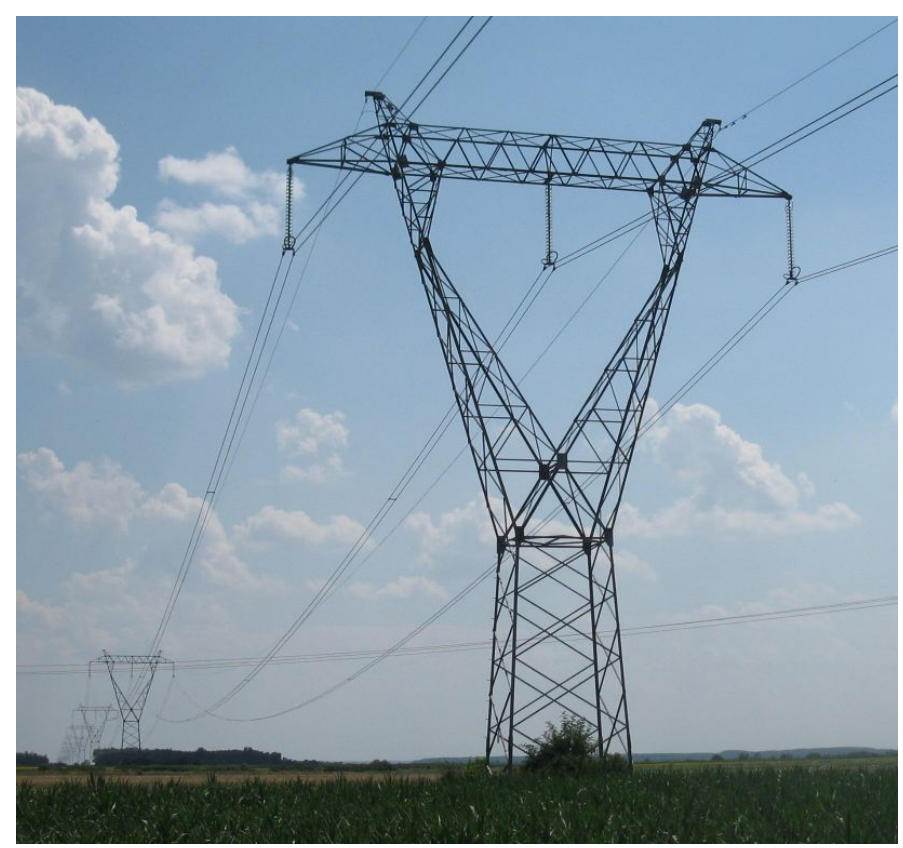

Fig. 1. High voltage overhead line

The existing professional literatures give a complete procedure for solving the equation of state and determination of the catenary constant [2]. However, a coordinate system which has an origin set at the vertex point of the conductor curve is generally in use, so the distance toward the left-hand or right-hand side support of the span is measured from the vertex, in both directions with a positive sign [7]. This method is mathematically not optimal for OHL design. For that reason this article uses a new coordinate system with an origin set on the line of the lefthand side support, on the elevation of the bottom of the lowerstanding support of the span. This way, the $y$-coordinate of the conductor curve presents the conductor height related to the $x$ axis, but its $x$-coordinate presents a horizontal distance from the
Electrical network designer, PhD student, Szivárvány u. 40., H-6500 Baja, Hungary

e-mail: hatibovic.alen@gmail.com 


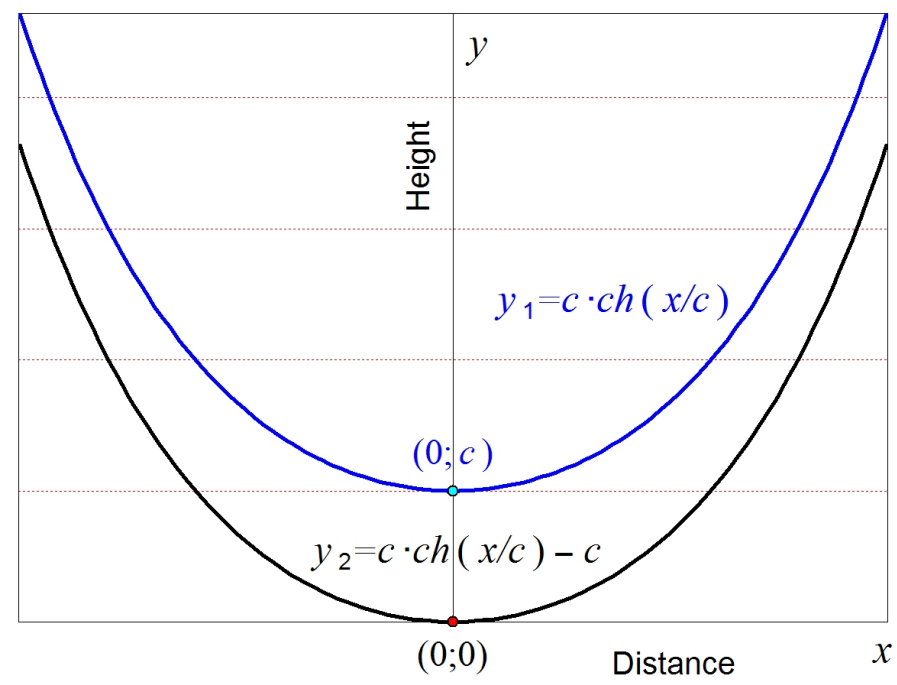

Fig. 2. Catenary curves

left-hand side support. In addition, this approach will help to recognize some mathematical similarities and differences easily between the catenary and parabola. This is one of the advantages of the new coordinate system applied in this article.

There is a basic condition in connection with the definition of the catenary, which says that the catenary constant should be positive $(c>0)$. The catenary curve $y_{1}$ shown in Figure 2 has the following equation:

$$
y_{1}=c \cdot \operatorname{ch}\left(\frac{x}{c}\right), \quad x \in(-\infty, \infty)
$$

Hence the vertex of the catenary curve $y_{1}$ is not located in the origin $(0 ; 0)$, as in the basic case of the parabola $\left(y=x_{2}\right)$, but it is in point $(0 ; c)$. If $y_{1}$ curve is replaced so that its vertex is set in the origin [1], the equation of the replaced curve $y_{2}$ is given by (2).

$$
y_{2}=c \cdot \operatorname{ch}\left(\frac{x}{c}\right)-c, \quad x \in(-\infty, \infty)
$$

In order to present the conductor curve in a mathematically convenient coordinate system for OHL design, the catenary curve $y_{2}$ has to be appropriately replaced both horizontally and vertically [6], as it is shown in Figure 3. The inclined span has been deliberately chosen instead of the levelled one, so the developed algorithm will be universal.

The following symbols are used in Figure 3

- $A\left(0 ; h_{1}\right)$ - left-hand side suspension point

- $B\left(S ; h_{2}\right)$ - right-hand side suspension point

- $\operatorname{MIN}\left(x_{M I N} ; y_{M I N}\right)$ - catenary's low point

- $C\left(x_{c} ; y_{c}\right)$-conductor's point with a maximal sag

- $S$ - span length

- $D_{\max }$ - maximal sag

- $y(x)$ - conductor curve (catenary)

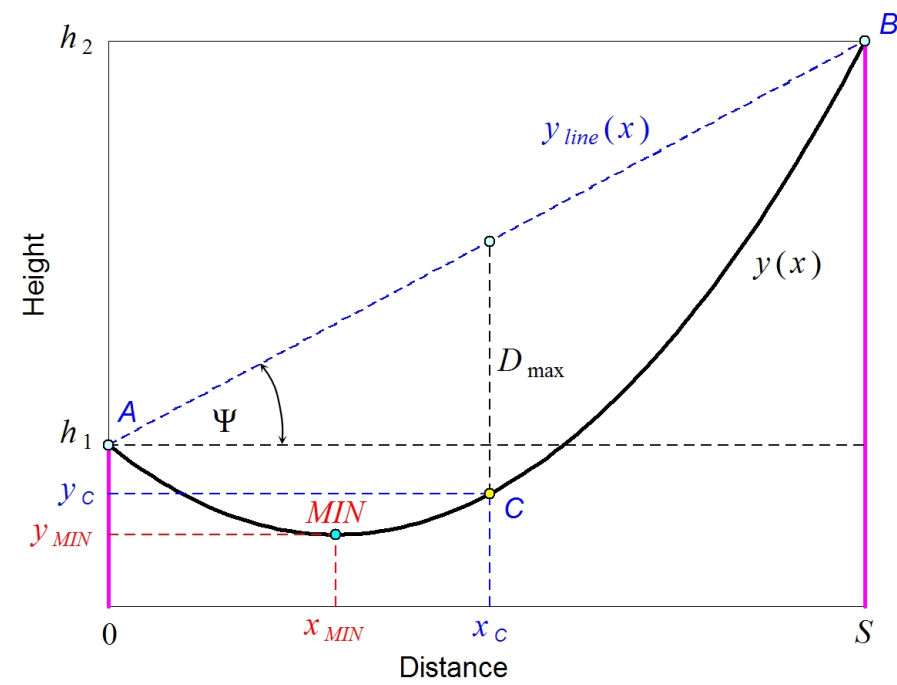

Fig. 3. Catenary curve in an inclined span

- $y_{\text {line }}(x)$ - straight line between the suspension points

- $\Psi$ - angle of the span inclination

The basic equation for the conductor curve in Figure 3 is the following in the interval $[0, S]$ :

$$
y(x)=c \cdot \operatorname{ch}\left(\frac{x-x_{M I N}}{c}\right)-c+y_{M I N}, \quad x \in[0, S]
$$

Another version of the previous equation is (4), but its exponential version is (5).

$$
\begin{gathered}
y(x)=2 c \cdot \operatorname{sh}^{2} \frac{x-x_{M I N}}{2 c}+y_{M I N}, \quad x \in[0, S] \\
y(x)=\frac{c}{2}\left(e^{\frac{x-x_{M I N}}{c}}+e^{-\frac{x-x_{M I N}}{c}}\right)-c+y_{M I N}, \quad x \in[0, S]
\end{gathered}
$$

All three equations are universal, i.e. they can be applied for inclined and levelled spans as well, but for a concrete usage the vertex point, i.e. the lowest point of the curve has to be determined previously. In comparison to the parabolic (quadratic) equation for the conductor curve there is a significant difference, since it can be defined even without knowing the vertex point of the conductor curve [4].

\section{Determination of the vertex point}

The coordinates of the catenary's vertex point can be determined on the basis of the following input data: $S, h_{1}, h_{2}, c$. By points $A$ and $B$ two equations in two unknowns can be written, then the first equation has to be subtracted from the second one.

$$
\begin{aligned}
h_{1} & =c \cdot \operatorname{ch} \frac{-x_{M I N}}{c}-c+y_{M I N} \\
h_{2} & =c \cdot \operatorname{ch} \frac{S-x_{M I N}}{c}-c+y_{M I N} \\
h_{2}-h_{1} & =c \cdot\left(\operatorname{ch} \frac{S-x_{M I N}}{c}-\operatorname{ch} \frac{-x_{M I N}}{c}\right)
\end{aligned}
$$


By the help of the identity (9] [8, 10] $x_{M I N}$ can be defined as (11).

$$
\begin{aligned}
\operatorname{ch}(x)-\operatorname{ch}(y) & =2 \operatorname{sh} \frac{x+y}{2} \operatorname{sh} \frac{x-y}{2} \\
h_{2}-h_{1} & =2 c \cdot \operatorname{sh} \frac{S-2 x_{M I N}}{2 c} \operatorname{sh} \frac{S}{2 c} \\
x_{M I N} & =\frac{S}{2}-c \cdot \operatorname{arsh} \frac{h_{2}-h_{1}}{2 c \cdot \operatorname{sh}(S / 2 c)}
\end{aligned}
$$

Using (11), the $y_{M I N}$ is obtained from (6) and transformed into 14 .

$$
\begin{aligned}
& y_{M I N}=h_{1}-c \cdot\left(\operatorname{ch} \frac{-x_{M I N}}{c}-1\right) \\
& y_{M I N}=h_{1}-2 c \cdot \operatorname{sh}^{2} \frac{x_{M I N}}{2 c} \\
& y_{M I N}=h_{1}-2 c \cdot \operatorname{sh}^{2}\left\{\frac{1}{2} \cdot\left[\frac{S}{2 c}-\operatorname{arsh} \frac{h_{2}-h_{1}}{2 c \cdot \operatorname{sh}(S / 2 c)}\right]\right\}
\end{aligned}
$$

Since the $x_{M I N}$ and $y_{M I N}$ are determined, the basic equation (4) for the conductor curve can be completed to (15), but the final catenary equation is given by the expression (16). The actual interval is $[0, S]$.

$$
\begin{array}{r}
y(x)=2 c \cdot \operatorname{sh}^{2} \frac{x-x_{M I N}}{2 c}+h_{1}-2 c \cdot \operatorname{sh}^{2} \frac{x_{M I N}}{2 c}, x \in[0, S] \\
y(x)=2 c \cdot\left(\operatorname{sh}^{2}\left\{\frac{1}{2 c}\left[x-\frac{S}{2}+c \cdot \operatorname{arsh} \frac{h_{2}-h_{1}}{2 c \cdot \operatorname{sh}(S / 2 c)}\right]\right\}-\right. \\
\left.\operatorname{sh}^{2}\left\{\frac{1}{2} \cdot\left[\frac{S}{2 c}-\operatorname{arsh} \frac{h_{2}-h_{1}}{2 c \cdot \operatorname{sh}(S / 2 c)}\right]\right\}\right)+h_{1}, \\
x \in[0, S]
\end{array}
$$

With the help of the previous equation the conductor height related to $x$-axis can be computed at any point of the span. Since the terrain within the span frequently differs from $x$-axis, the height of the terrain related to $x$-axis has to be taken into consideration when computing the ground clearance of the OHL. Another important usage of the equation (16) is drawing the conductor curve.

The vertex point of the catenary is generally identical to the lowest point of the conductor. However, there are special cases of inclined spans when the vertex is out of the span and hence differs from the lowest point of the conductor. At such times the latter point is identical to the lower suspension point of the span, but the coordinates of the catenary's vertex are still given by (11), (14). However, the equation (16) is applicable in any case, so it proves its universality.

\section{Sag equation and characteristic sags}

\subsection{Derivation of the Sag Equation}

The conductor sag is the distance measured vertically from the conductor to the straight line (chord) joining two suspension points of a span. Actually the sag varies in the interval of the

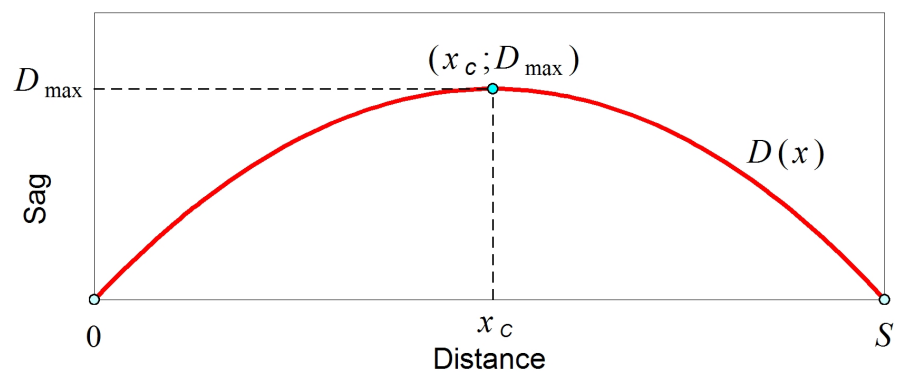

Fig. 4. Sag curve

span, i.e. increases from zero to maximum then decreases to zero, going from the left-hand side support to the right-hand side one. It can be appropriately described by the sag equation $D(x)$ as the function of $x$, where $x$ varies from zero to the span length, $x \in[0, S]$.

By the use of the equation for the conductor curve, the equation for the sag curve (shortly called as sag equation) can also be obtained. It is necessary for the determination of the maximal sag location $\left(x_{c}\right)$ within the span and its value $\left(D_{\max }\right)$. In order to obtain that, first the equation of the straight line ( $\left.y_{\text {line }}\right)$ passing through the suspension points $A$ and $B$ has to be defined in the interval $[0, S]$, than subtract (3). The result provided is the sag equation $D(x)$, which is usable for the sag calculation at any point of the span.

$$
\begin{array}{r}
y_{\text {line }}=\frac{h_{2}-h_{1}}{S}+h_{1}, \quad x \in[0, S] \\
D(x)=y_{\text {line }}(x)-y(x), \quad x \in[0, S]
\end{array}
$$

$$
\begin{aligned}
D(x)=\frac{h_{2}-h_{1}}{S} x+h_{1}- & \\
c & \cdot \operatorname{ch} \frac{x-x_{M I N}}{c}+c-y_{M I N}, \quad x \in[0, S]
\end{aligned}
$$

The sag curve is shown in Figure 4 Differently to the conductor curve it has a maximum point.

\subsection{Location of the maximal sag}

Finding the first derivative of (19) and then solving the equation (21), the maximal sag location $\left(x_{c}\right)$ within the span is obtained, then given by (24).

$$
\begin{aligned}
& \frac{d D(x)}{d x}=\frac{h_{2}-h_{1}}{S}-\operatorname{sh} \frac{x-x_{M I N}}{c} \\
& \frac{d D(x)}{d x}=0 \Rightarrow x_{c} \\
& \frac{h_{2}-h_{1}}{S}-\operatorname{sh} \frac{x_{c}-x_{M I N}}{c}=0 \\
& x_{c}=x_{M I N}+c \cdot \operatorname{arsh} \frac{h_{2}-h_{1}}{S} \\
& x_{c}=\frac{S}{2}+c \cdot\left[\operatorname{arsh} \frac{h_{2}-h_{1}}{S}-\operatorname{arsh} \frac{h_{2}-h_{1}}{2 c \cdot \operatorname{sh}(S / 2 c)}\right]
\end{aligned}
$$


From 24 it is obvious that the maximal sag of the catenary in an inclined span is not located at a mid-span, but it is moved toward one of the two suspension points. Now there is a question whether it is moved toward the higher or the lower one. The answer to this question will be given here without the use of numerical computation.

Denoting the second summand in 24 by $q$ it gives the expression 25):

$$
x_{c}=\frac{S}{2}+q
$$

Now let us assume that the maximal sag is moved from the mid-span toward the higher suspension point and that the righthand side one is higher than the left-hand side one, i.e. assume that relation 26 is valid and then check it mathematically step by step.

$$
\text { if } h_{1}<h_{2} \Rightarrow q>0
$$

The initial conditions are given: $S>0, c>0, h_{1}>0, h_{2}>0$. The main steps for checking the validity of the assumption given by 26 are shown in the following lines:

$$
\begin{gathered}
c \cdot\left[\operatorname{arsh} \frac{h_{2}-h_{1}}{S}-\operatorname{arsh} \frac{h_{2}-h_{1}}{2 c \cdot \operatorname{sh}(S / 2 c)}\right]>0 \\
\operatorname{arsh} \frac{h_{2}-h_{1}}{S}>\operatorname{arsh} \frac{h_{2}-h_{1}}{2 c \cdot \operatorname{sh}(S / 2 c)}
\end{gathered}
$$

The inverse hyperbolic sine is a monotonic, strictly increasing function [9], so

$$
\text { if } \quad x_{2}>x_{1} \Rightarrow \operatorname{arsh}\left(x_{2}\right)>\operatorname{arsh}\left(x_{1}\right)
$$

Applying 29, in 28, gives (30) which can deduce (32)

$$
\begin{aligned}
\frac{h_{2}-h_{1}}{S} & >\frac{h_{2}-h_{1}}{2 c \cdot \operatorname{sh}(S / 2 c)} \\
\frac{1}{S} & >\frac{1}{2 c \cdot \operatorname{sh}(S / 2 c)} \\
S / 2 c & <\operatorname{sh}(S / 2 c)
\end{aligned}
$$

Since $S / 2 c>0$ and taking into the consideration relation (33), the previous one is valid.

$$
\text { if } \quad x>0 \Rightarrow x<\operatorname{sh}(x)
$$

In this way the validity of the assumption (26) is also proved. The same process applied for cases $h_{1}>h_{2}$ and $h_{1}=h_{2}$ gives further two relations:

$$
\begin{array}{llll}
\text { if } & h_{1}>h_{2} & \Rightarrow & q<0 \\
\text { if } & h_{1}=h_{2} \quad & \Rightarrow & q=0
\end{array}
$$

Thus the above question of the movement of $D_{\max }$ has been satisfactory answered. The previous relation refers to a levelled span when there is no movement of $D_{\max }$.
Summarizing 26, 34 and 35 the final conclusion of the location of $D_{\max }$ related to a mid-span, proved mathematically here, is the following:

The maximal sag of the catenary conductor curve in a levelled span is located at a mid-span, but in an inclined span it is moved from a mid-span toward a higher suspension point.

This is one essential difference in comparison to the parabola, since the maximal sag of the parabolic conductor curve is always located at a mid-span, both in levelled and inclined spans as well [3]. This feature effectively simplifies the parabola based algorithms for overhead line design.

\subsection{Characteristic sags}

Putting $x_{c}$ into a sag equation the maximal sag is obtained. The main steps of the deduction are the following:

$$
D_{\text {max }}=D\left(x_{c}\right)=y_{\text {line }}\left(x_{c}\right)-y\left(x_{c}\right)
$$

$$
\begin{aligned}
D_{\text {max }}= & \frac{h_{2}-h_{1}}{S} x_{c}+h_{1}-c \cdot \operatorname{ch} \frac{x_{c}-x_{M I N}}{c}+c-y_{M I N} \\
D_{\text {max }}= & 2 c \cdot\left(\frac{h_{2}-h_{1}}{2 S}\left[\frac{S}{2 c}-\operatorname{arsh} \frac{h_{2}-h_{1}}{2 c \cdot \operatorname{sh}(S / 2 c)}+\operatorname{arsh} \frac{h_{2}-h_{1}}{S}\right]-\right. \\
& \left.\operatorname{sh}^{2}\left(\frac{1}{2} \operatorname{arsh} \frac{h_{2}-h_{1}}{S}\right)+\operatorname{sh}^{2}\left\{\frac{1}{2} \cdot\left[\frac{S}{2 c}-\operatorname{arsh} \frac{h_{2}-h_{1}}{2 c \cdot \operatorname{sh}(S / 2 c)}\right]\right\}\right)
\end{aligned}
$$

The previous expression is a formula for calculating the maximal sag of the catenary conductor curve in an inclined span. Similarly, formulas 39) and 40, for the other characteristic sags can be defined by the use of the basic sag equation (19).

Mid-span sag:

$$
\begin{array}{r}
D(S / 2)=\frac{h_{2}-h_{1}}{2}-2 c \cdot\left(\operatorname{sh}^{2}\left[\frac{1}{2} \operatorname{arsh} \frac{h_{2}-h_{1}}{2 c \cdot \operatorname{sh}(S / 2 c)}\right]-\right. \\
\left.\operatorname{sh}^{2}\left\{\frac{1}{2} \cdot\left[\frac{S}{2 c}-\operatorname{arsh} \frac{h_{2}-h_{1}}{2 c \cdot \operatorname{sh}(S / 2 c)}\right]\right\}\right)
\end{array}
$$

Sag at the lowest point of the conductor:

$$
\begin{array}{r}
D\left(x_{M I N}\right)=2 c \cdot\left(\frac{h_{2}-h_{1}}{2 S}\left[\frac{S}{2 c}-\operatorname{arsh} \frac{h_{2}-h_{1}}{2 c \cdot \operatorname{sh}(S / 2 c)}\right]+\right. \\
\left.\operatorname{sh}^{2}\left(\frac{1}{2} \cdot\left\{\frac{S}{2 c}-\operatorname{arsh} \frac{h_{2}-h_{1}}{2 c \cdot \operatorname{sh}(S / 2 c)}\right\}\right)\right), \\
\forall 0 \leq x_{M I N} \leq S
\end{array}
$$

\section{Levelled spans}

In a levelled span the suspension points are on the same elevation $\left(h_{1}=h_{2}=h\right)$. Actually, it is a simplification of an inclined span. In this special case the equations for the conductor curve (41), (42), 43) are simpler than the adequate ones in an inclined span, since the lowest point (44) of the conductor is located at a mid-span. 


$$
\begin{gathered}
y(x)=c \cdot\left(\operatorname{ch} \frac{x-S / 2}{c}-\operatorname{ch} \frac{S}{2 c}\right)+h, \quad x \in[0, S] \\
y(x)=2 c \cdot\left(\operatorname{sh}^{2} \frac{x-S / 2}{2 c}-\operatorname{sh}^{2} \frac{S}{4 c}\right)+h, \quad x \in[0, S] \\
y(x)=\frac{c}{2} \cdot\left[\left(e^{\frac{x-S / 2}{2 c}}-e^{-\frac{x-S / 2}{2 c}}\right)^{2}-\left(e^{\frac{S}{4 c}}-e^{-\frac{S}{4 c}}\right)^{2}\right]+h, \quad x \in[0, S] \\
\operatorname{MIN}\left(\frac{S}{2} ; h-2 c \cdot \operatorname{sh}^{2} \frac{S}{4 c}\right)
\end{gathered}
$$

The equation for the conductor sag in a levelled span is given by the expressions 45, 46 or 47, but the formula for the calculation of the maximal sag is (48).

$$
\begin{gathered}
D(x)=c \cdot\left(\operatorname{ch} \frac{S}{2 c}-\operatorname{ch} \frac{x-S / 2}{c}\right), \quad x \in[0, S] \\
D(x)=2 c \cdot\left(\operatorname{sh}^{2} \frac{S}{4 c}-\operatorname{sh}^{2} \frac{x-S / 2}{2 c}\right), \quad x \in[0, S] \\
D(x)=\frac{c}{2} \cdot\left[\left(e^{\frac{S}{4 c}}-e^{-\frac{S}{4 c}}\right)^{2}-\left(e^{\frac{x-S / 2}{2 c}}-e^{-\frac{x-S / 2}{2 c}}\right)^{2}\right], x \in[0, S] \\
D_{\text {max }}=D(S / 2)=D\left(x_{M I N}\right)=2 c \cdot \operatorname{sh}^{2} \frac{S}{4 c}
\end{gathered}
$$

\section{Future work}

Complementing the existing knowledge, the shown formulas provided in the new coordinate system give an opportunity for further important research and mathematical analysis useful for precise OHL design and more efficient education of the electrical network designers. There is a possibility for the further works: finding the relationship between sags in levelled and inclined spans, a parabolic approximation of the catenary in inclined spans, inclined span modelling by a given levelled span, etc.

\section{Conclusions}

Beside the determination of the new equations for the conductor and sag curves some characteristic differences between the catenary and parabola have been revealed. Pre-eminently it should be mentioned that the method referring to defining the equation for the conductor curve significantly differs in the case of parabolic and catenary approach. While for the catenary the coordinates of the vertex point are necessary initial data, for parabola they are not.

It has been shown that by the use of the new catenary equation the direction of the catenary's maximal sag movement from the mid-span can be determined with symbolic computation.

By the help of the basic sag equation provided in the article for sag calculation at an arbitrary point of a span, the special sag formulas are obtained for computing of characteristic sags: maximal sag, mid-span sag and low point sag. On the basis of the presented catenary sag formulas the last three sags are different in an inclined span, but they are all equal in a levelled span.

The following very important feature of the catenary is identified: the sag function of its curve replaced from interval $[0, S]$ to $[-S / 2, S / 2]$ is an even function in case of a levelled span, but in an inclined span it is neither an even nor odd function. Comparing it to the parabola this is another special difference, since the adequate feature of the latter function says [5]: the parabolic (quadratic) sag function of its curve replaced from interval $[0, S]$ to $[-S / 2, S / 2]$ is an even function both in case of levelled and inclined spans.

\section{References}

1 Sag-Tension Calculation Methods for Overhead Lines, CIGRÉ Technical Brochure No. 324, (2007). CIGRÉ WG B2-12.

2 Douglass DA, Thrash R, Sag and Tension of Conductor, Taylor \& Fransis Group LLC., 2006.

3 Hatibovic A, Determination of the Lowest Point of the Conductor in Inclined Spans Based on a Known Maximal Sag of the Parabola, CIRED 22nd International Conference on Electricity Distribution, (2013), 1-4, DOI $10.1049 / \mathrm{cp} .2013 .0583$

4 Hatibovic A, Integral Calculus Usage for Conductor Length Determination on the Basis of Known Maximal Sag of a Parabola, Periodica Polytechnica Electrical Engineering, 56(2), (2012), DOI 10.3311/PPee.7076

5 Hatibovic A, Parabolic Equation for Conductor Sag in Levelled and Inclined Spans, 13th International Conference on Energetics-Electrical Engineering, (2012), 84-89.

6 Larson R, Hosteler R, Edwards BH, Essential Calculus Early Transcendental Functions, Houghton Mifflin Company, 2008.

7 Rashid MH, Power Electronics Handbook, Elsevier, 2011.

8 Weir MD, Hass J, Thomas' Calculus, Pearson, 2010.

9 http://adattar.vmmi.org/konyvek/220/03_halmazok,_relaciok, -fuggvenyek.pdf

10 http://perso.univ-rennes1.fr/maximilian.bauer/AN1/ fonctions_hyperboliques.pdf 